\title{
Activity Code
}

National Cancer Institute

\section{Source}

National Cancer Institute. Activity Code. NCI Thesaurus. Code C60756.

The designated category assigned by the National Institutes of Health to a specific funding mechanism for an extramural research activity. 